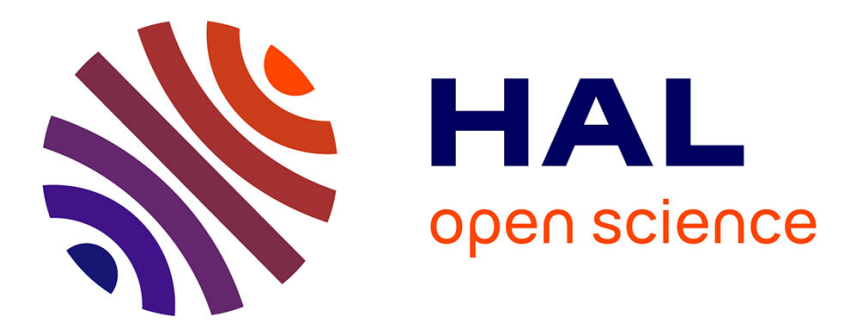

\title{
Stabilization of nonlinear switched systems using control Lyapunov functions
}

Emmanuel Moulay, Romain Bourdais, Wilfrid Perruquetti

\section{To cite this version:}

Emmanuel Moulay, Romain Bourdais, Wilfrid Perruquetti. Stabilization of nonlinear switched systems using control Lyapunov functions. Nonlinear Analysis: Hybrid Systems, 2007, 1 (4), pp.482-490. 10.1016/j.nahs.2005.12.001 . hal-03088747

\section{HAL Id: hal-03088747 https://hal.science/hal-03088747}

Submitted on 19 Jan 2021

HAL is a multi-disciplinary open access archive for the deposit and dissemination of scientific research documents, whether they are published or not. The documents may come from teaching and research institutions in France or abroad, or from public or private research centers.
L'archive ouverte pluridisciplinaire HAL, est destinée au dépôt et à la diffusion de documents scientifiques de niveau recherche, publiés ou non, émanant des établissements d'enseignement et de recherche français ou étrangers, des laboratoires publics ou privés. 


\title{
Stabilization of nonlinear switched systems using control Lyapunov functions *
}

\author{
Emmanuel MOULAY *,a,b, Romain BOURDAIS **,a, \\ Wilfrid PERRUQUETTI $* * *$, a \\ ${ }^{a}$ LAGIS (UMR-CNRS 8146), Ecole centrale de Lille, Cité Scientifique, 59651 \\ Villeneuve d'Ascq Cedex, France \\ ${ }^{\mathrm{b}}$ Laboratoire de Mathématiques Paul Painlevé (UMR-CNRS 8524), Université de \\ Sciences et Technologies de Lille
}

\begin{abstract}
In this paper, we study the stabilization of general nonlinear switched systems by using control Lyapunov functions. The concept of control Lyapunov function for nonlinear control systems is generalized to switched control systems. The first part of our contribution deals with a necessary and sufficient condition of stabilization. The main idea is to use a common control Lyapunov function, this is achieved with the converse Lyapunov theorem dedicated to switched systems. In a second part, an explicit construction of a common control Lyapunov function is addressed to a finite family of switched systems. The approach uses a family of control Lyapunov functions attached to the subsystems.
\end{abstract}

Key words: Stabilization, Nonlinear switched systems, control Lyapunov function.

\section{Introduction}

Current interest in intelligent control has led to the development of the study of switched systems. Informally, a switched system is an indexed family of

ऋ Partially supported by programs ARCIR-ROBOCOOP $\mathbf{5 3}$

* (33) +3 20335450

**(33) +320676032

**(33) +3 20335417

Email addresses: emmanuel.moulay@ec-lille.fr (Emmanuel MOULAY ), bourdais.romain@ec-lille.fr (Romain BOURDAIS ), wilfrid.perruquetti@ec-lille.fr (Wilfrid PERRUQUETTI ). 
continuous-time and a rule that determines the switching between them. As a survey on the subject, the paper [1] presents the recent results, and lots of open problems may be studied. Many papers appear in the last decade dedicated to the stability of hybrid dynamical systems : [2], [3], [4]. The problem of perturbed switched systems has been addressed in [5], using time dependant varying control. Our paper is based on the paper of J.L. Mancilla-Aguillar [6] which deals with the stability of general nonlinear switched systems. We use its converse theorem to treat the problem of stabilization of nonlinear switched systems. In [7], an other converse theorem dedicated to pairwise commuting asymptotically stable systems appears. Even if the results are more constructive, the class of systems studied in [7] is included in the one studied in $[6]$.

In this paper, the switching signal is imposed, typically a system controlled by an exterior operator as a driver and its gearbox. If it is controllable, it is rather easy to obtain the stabilization of switched control systems by using the fact that a control Lyapunov function for a subsystem is also a control Lyapunov function for the global switched system (see [8]).

The outline of the paper is as follows. In section 2, we recall the basic notations and definitions which will be used. In section 3, we present a necessary and sufficient condition for the stabilization issue. Then, in section 4 , we give an explicit formula of the control law, by using the Lin-Sontag feedback control. To ensure the stabilization, we need to find a common control Lyapunov function. In section 5 , we propose a constructive method of this function, by using a family of control Lyapunov functions attached to the subsystems. We make concluding remarks and sketch some directions for future research in section 6.

\section{Notations and Definitions}

We first introduce some notations and definitions for subsequent use. $\mathbb{R}^{n}$ denotes the usual $n$-dimensional Euclidean space and $\|\cdot\|_{n}$ its Euclidean norm. In the following, $\mathcal{V}$ will be a neighborhood of the origin in $\mathbb{R}^{n}$.

We assume that a subset $A$ of a topological space is given the induced subset topology, and we use $\bar{A}$ to denote its closure, $\stackrel{\circ}{A}$ its interior, $\partial A=\bar{A} \backslash \stackrel{\circ}{A}$ its boundary. Let $E, F$ be topological spaces and $p: E \rightarrow F$ a continuous map. The map is called continuous proper if for every compact set $K \subseteq F$, the preimage $p^{-1}(K)$ is compact in $E$.

Let $V: \mathcal{V} \rightarrow \mathbb{R}_{\geq 0}$ be a continous function and $c>0$, we define the set

$$
I_{c}=\{x \in \mathcal{V}: V(x) \leq c\}
$$


We denote with $C^{0}\left(\mathbb{R}^{n}\right)$ the class of continuous vector fields on $\mathbb{R}^{n}$, equipped with the topology of uniform convergence on compact sets, $C^{1}\left(\mathbb{R}^{n}\right)$ the class of differentiable functions with continuous partial derivatives. $L_{l o c}^{\infty}\left(\mathbb{R}^{n}\right)$ is the set of measurable functions $f: \mathbb{R}^{n} \rightarrow \mathbb{R}^{n}$ such that for all compact set $K$ of $\mathbb{R}^{n}$, there exists $C_{K}>0$ such that $\|f(x)\|<C_{K}$ a.e. in $K . \epsilon \mathcal{B}^{n}$ denotes the open ball centered at the origin of radius $\epsilon$ in $\mathbb{R}^{n}$. $\mathcal{B}^{n}$ is the unit open ball, and $\dot{\mathcal{B}}^{n}=\mathcal{B}^{n} \backslash\{0\}$.

A continuous function $\alpha:[0, a] \rightarrow \mathbb{R}_{\geq 0}$ belongs to class $\mathcal{K}$ if it is strictly increasing and $\alpha(0)=0$. It is said to belong to class $\mathcal{K}_{\infty}$ if $a=+\infty$ and $\alpha(r) \rightarrow+\infty$ as $r \rightarrow+\infty$.

Let us consider the $C^{1}$-function $\gamma: \mathbb{R}^{n} \rightarrow \mathbb{R}$, then $\nabla_{j} \gamma$ denotes the $j$-th component of the gradient of $\gamma$.

A set-valued function $\Phi$ from $\mathcal{X}$ to $\mathcal{Y}$ is a function that maps $x \in \mathcal{X}$ to a set $\Phi(x) \subset \mathcal{Y}$. Let $\mathcal{X}$ and $\mathcal{Y}$ be two vector spaces and $\Phi: \mathcal{X} \rightarrow \mathcal{Y}$ a set-valued function, $\Phi$ is lower semi-continuous if $\{x \in \mathcal{X}: \Phi(x) \cap O \neq \emptyset\}$ is open in $\mathcal{X}$ for every open set $O \subset \mathcal{Y}$. $\Phi$ is locally Lipschitz if for any $x_{0} \in \mathcal{X}$, there exists a neighborhood $\mathcal{N}\left(x_{0}\right) \subset \mathcal{X}$ and a constant $l \geq 0$ such that for all $x, x^{\prime} \in \mathcal{N}\left(x_{0}\right)$,

$$
\Phi(x) \subset \Phi\left(x^{\prime}\right)+l\left\|x-x^{\prime}\right\|_{\mathcal{X}} \mathcal{B}^{\mathcal{Y}}
$$

where $\mathcal{B}^{\mathcal{Y}}$ is the unit ball in $\mathcal{Y}$ and $\|.\|_{\mathcal{X}}$ is the norm on $\mathcal{X}$. Let $\Gamma$ be an index set.

Definition $1 \mathcal{P}(\Gamma)$ is the set $\left\{f_{\sigma} \in C^{0}\left(\mathbb{R}^{n}\right): \sigma \in \Gamma\right\}$ such that,

(1) $\mathcal{P}(\Gamma)$ is equibounded, i.e. $\sup _{\sigma \in \Gamma}\left\|f_{\sigma}(x)\right\|_{n}<+\infty$ for all $x \in \mathbb{R}^{n}$,

(2) $\mathcal{P}(\Gamma)$ is uniformly locally Lipschitz, i.e. for each $\delta \in \mathbb{N}$, there exists $l_{\delta} \geq 0$ such that

$$
\left\|f_{\sigma}(x)-f_{\sigma}(y)\right\|_{n} \leq l_{\delta}\|x-y\|_{n}
$$

for all $(x, y) \in \delta \mathcal{B}^{n} \times \delta \mathcal{B}^{n}$ and all $\sigma \in \Gamma$.

$\dot{\mathcal{P}}(\Gamma)$ is the set $\left\{f_{\sigma} \in C^{0}\left(\mathbb{R}^{n} \backslash\{0\}, \mathbb{R}^{n}\right) \cap L_{l o c}^{\infty}\left(\mathbb{R}^{n}\right): \sigma \in \Gamma\right\}$ such that,

(1) $\dot{\mathcal{P}}(\Gamma)$ is equibounded,

(2) $\dot{\mathcal{P}}(\Gamma)$ is uniformly locally Lipschitz outside the origin, i.e. for each $\delta \in \mathbb{N}$, there exists $l_{\delta} \geq 0$ such that

$$
\left\|f_{\sigma}(x)-f_{\sigma}(y)\right\|_{n} \leq l_{\delta}\|x-y\|_{n}
$$

for all $(x, y) \in \delta \dot{\mathcal{B}}^{n} \times \delta \dot{\mathcal{B}}^{n}$ and all $\sigma \in \Gamma$.

Let us recall the classical definition of switching signal. Given a nonempty set $C$, we say that a function $s: \mathbb{R}_{\geq 0} \rightarrow C$ is a $C$-switching signal if $s: \mathbb{R}_{\geq 0} \rightarrow C$ is a piecewise constant function, i.e. the set of points where the function $s$ has jumps is a discrete set, and $s$ is constant between jumps. We do not take in 
consideration the accumulation points to avoid a Zeno phenomena (for more information on this point, see [5]). In what follows, we will denotes by $\mathcal{S}(C)$ the family of all $C$-switching signal.

Let us consider the system

$$
\dot{x}=f_{s}(x), \quad x \in \mathbb{R}^{n}, s \in \mathcal{S}(\Gamma)
$$

where $f_{s} \in \mathcal{P}(\Gamma)$ and $f_{s}(0)=0$, for all $s \in \mathcal{S}(\Gamma)$. Associated with each $s \in \mathcal{S}(\Gamma)$, there exists a sequence of real numbers $0=t_{0}<t_{1}<\ldots<t_{k}<\ldots$ and a sequence of indexes $\sigma_{0}, \sigma_{1}, \ldots, \sigma_{k}, \ldots$ such that $s(t)=\sigma_{k}$ for all $t_{k} \leq$ $t<t_{k+1}$. A solution of (1) starting from $\xi \in \mathbb{R}^{n}$ is an absolutely continuous function $x:[0, T) \rightarrow \mathbb{R}^{n}$ such that $x(0)=\xi$, and for all $k \in \mathbb{N}$,

$$
\dot{x}(t)=f_{\sigma_{k}}(x(t))
$$

a.e. in $\left[t_{k}, t_{k+1}\right) \cap[0, T)$. Due to the local Lipschitz property of the elements of $\mathcal{P}(\Gamma)$, for each $s \in \mathcal{S}(\Gamma)$ and for each initial condition $\xi \in \mathbb{R}^{n}$ there exists a unique maximally defined solution denoted by $x(t, \xi, s)$.

As usual, the system (1) is asymptotically stable if

(1) the system (1) is stable : i.e. for all $\epsilon>0$ there exists $\delta>0$ such that for all $\xi \in \delta \mathcal{B}^{n}, x(t, \xi, s) \in \epsilon \mathcal{B}^{n}$ for all $s \in \mathcal{S}(\Gamma)$,

(2) $\lim _{t \rightarrow+\infty} x(t, \xi, s)=0$ for all $s \in \mathcal{S}(\Gamma)$.

Definition 2 [6] A common Lyapunov function $V$ for $\mathcal{P}(\Gamma)$ is a $C^{1}$ proper function $V: \mathbb{R}^{n} \rightarrow \mathbb{R}_{\geq 0}$ such that there exist two functions $\alpha_{1}$ and $\alpha_{2}$ of class $\mathcal{K}$ and a continuous positive definite function $\alpha_{3}$ that verify:

(1) $\alpha_{1}\left(\|x\|_{n}\right) \leq V(x) \leq \alpha_{2}\left(\|x\|_{n}\right), \quad \forall x \in \mathcal{V}$,

(2) $\left\langle\nabla V(x), f_{s}(x)\right\rangle \leq-\alpha_{3}\left(\|x\|_{n}\right), \quad \forall x \in \mathcal{V}, \forall s \in \mathcal{S}(\Gamma)$.

Remark 3 If the common Lyapunov function is globally defined on $\mathbb{R}^{n}$, in addition we suppose that $\alpha_{1}, \alpha_{2} \in \mathcal{K}_{\infty}$. The common Lyapunov function is said to be decrescent. This ensures that $\lim _{\|x\|_{n} \rightarrow+\infty} V(x)=+\infty$.

Let us consider the control system

$$
\dot{x}=f_{s}(x, u), \quad x \in \mathbb{R}^{n}, u \in \overline{\mathcal{B}^{m}}, s \in \mathcal{S}(\Gamma) .
$$

Definition 4 Let $s \in \mathcal{S}(\Gamma)$, the system (2) is weakly stabilizable (respectively stabilizable) if there exists $u_{s} \in \dot{\mathcal{P}}(\Gamma)$ (respectively $u_{s} \in \mathcal{P}(\Gamma)$ ) such that the closed-loop system

$$
\dot{x}=f_{s}\left(x, u_{s}(x)\right)
$$

is asymptotically stable. 
Remark 5 For the weakly stabilization case, the closed-loop system is discontinuous at the origin. This problem can be solved, as it is shown in [9]. Indeed, the closed-loop system can be extended to an asymptotically continuous one, because of the autonomous property. The fact that $u_{s}$ may fail to be even continuous at the origin for all $s \in \mathcal{S}(\Gamma)$ causes no problems regarding uniqueness of solutions, as it is easy to verify from the definition of asymptotic stability.

Remark 6 In this paper, we will consider, with no loss of generality the closed unit ball $\overline{\mathcal{B}^{m}}$. Indeed, any compact set can be included in a closed ball, and any closed ball is topologically equivalent to the unit closed ball. So our results are also true for any compact set.

Definition $7 A C^{1}$ proper positive definite function $V: \mathcal{V} \rightarrow \mathbb{R}_{>0}$ is said to be a common control Lyapunov function for the system (2) if for all $x \in \mathcal{V} \backslash\{0\}$

$$
\min _{u \in \overline{\mathcal{B}}^{m}}\left\langle\nabla V(x), f_{s}(x, u)\right\rangle<0, \quad \forall s \in \mathcal{S}(\Gamma)
$$

Such a control Lyapunov function satisfies the small control property if for each $0<\epsilon \leq 1$, there exists $\delta>0$ such that, if $x \in \delta \mathcal{B}^{n} \subset \mathcal{V}$, then for all $s \in \mathcal{S}(\Gamma)$, there exists some $u_{s} \in \epsilon \mathcal{B}^{m}$ such that

$$
\left\langle\nabla V(x), f_{s}(x, u)\right\rangle<0
$$

\section{A necessary and sufficient condition for the stabilization's prob- lem}

In this section, we first recall the main results on the stability of switched systems.

Theorem 8 [6] The system (1) is asymptotically stable if and only if there exists a common Lyapunov function for $\mathcal{P}(\Gamma)$ where $\mathcal{P}(\Gamma)$ is defined in Definition 1.

Let us recall the fundamental theorem of Mickael which is in $[10$, Theorem 9.5.3] and [11].

Theorem 9 (of Mickael) Let $\mathcal{X}$ be a compact Hausdorff space and $\mathcal{Y}$ a $B a$ nach space, for every lower semi-continuous (respectively locally Lipschitz) set-valued function $\Phi: \mathcal{X} \rightarrow 2^{\mathcal{Y}}, x \mapsto \Phi(x)$ where $2^{\mathcal{Y}}$ will denote the family of non-empty, closed, convex subsets of $\mathcal{Y}$, it is possible to extract a continuous (respectively locally Lipschitz) function $f$ such that $f(x) \in \Phi(x)$ for all $x \in \mathcal{X}$. 
In order to use the Mickael's theorem, we introduce the affine system :

$$
\dot{x}=f_{s}(x)+\left\langle g_{s}(x), u\right\rangle, \quad x \in \mathbb{R}^{n}, u \in \mathbb{R}^{m}, s \in \mathcal{S}(\Gamma)
$$

where $f_{s}: \mathbb{R}^{n} \rightarrow \mathbb{R}, g_{s}: \mathbb{R}^{n} \rightarrow \mathbb{R}^{m}$ are in $\mathcal{P}(\Gamma)$ and $f_{s}(0)=0$ for all $s \in \mathcal{S}(\Gamma)$. Let us introduce some more notations. For a $C^{1}$ positive definite function $V$, we will denote with

$$
\begin{aligned}
a_{s}(x) & =\left\langle\nabla V(x), f_{s}(x)\right\rangle, \\
B_{s}(x) & =\left\langle\nabla V(x), g_{s}(x)\right\rangle \in \mathbb{R}^{m}, \\
b_{s} & =\left\|B_{s}(x)\right\|_{m}^{2} .
\end{aligned}
$$

Remark 10 If $m=1$, the small control property is equivalent to

$$
\limsup _{\|x\|_{n} \rightarrow 0} \frac{a_{s}(x)}{\left|B_{s}(x)\right|} \in \mathbb{R}_{\leq 0} \cup\{-\infty\}
$$

for all $s \in \mathcal{S}(\Gamma)$.

The main result of this paper may be stated as follows:

Theorem 11 The system (5) is weakly stabilizable if and only if there exists a common control Lyapunov function $V$ for the system (5).

In addition, if $V$ satisfies the small control property, the system (5) is stabilizable. Moreover, the system (5) is globally stabilizable if and only if $V$ is globally defined.

Proof. If the control system (5) is weakly stabilizable, then the closed-loop system

$$
\dot{x}=f_{s}(x)+\left\langle g_{s}(x), u_{s}(x)\right\rangle
$$

is asymptotically stable. This system which can be discontinuous at the origin can be extended to an equivalent one $\left(S_{e q}\right)$ continous everywhere (see [9]). By using Theorem 8, there exists a common Lyapunov function $V$ for the system $\left(S_{e q}\right)$ which is also a common Lyapunov function $V$ for the closed-loop system (6). Then, $V$ is a common control Lyapunov function for the system (5).

Conversely, suppose that there exists a common control Lyapunov function $V: \mathcal{V} \rightarrow \mathbb{R}_{\geq 0}$ for the system (5). We have

$$
\inf _{u \in \mathbb{R}^{m}}\left\langle\nabla V(x), f_{s}(x, u)\right\rangle \leq \min _{u \in \overline{\mathcal{B}}^{m}}\left\langle\nabla V(x), f_{s}(x, u)\right\rangle<0, \quad \forall s \in \mathcal{S}(\Gamma) .
$$

Let $s \in \mathcal{S}(\Gamma)$, then we introduce the set valued function $\Phi_{s}$ defined for $x \in$ $I_{c} \backslash\{0\}$ by

$$
\Phi_{s}(x)=\left\{v \in \mathbb{R}^{m}: a_{s}(x)+\left\langle B_{s}(x), v\right\rangle<0\right\} .
$$

As $v \mapsto a_{s}(x)+\left\langle B_{s}(x), v\right\rangle$ is affine, it implies that for all $x \in I_{c} \backslash\{0\}, \Phi_{s}(x)$ belongs to the family of closed convex subsets of $\mathbb{R}^{m}$. As $f_{s}$ and $g_{s}$ belong to the class $\mathcal{P}(\Gamma)$ and $V$ is a $C^{1}$-function, $a_{s}(x)+\left\langle B_{s}(x), v\right\rangle$ is locally Lipschitz 
for all $x \in I_{c} \backslash\{0\}$. We apply the theorem 9 of Mickael to find a function $u_{s}$ locally Lipschitz on $x \in I_{c}$ such that $u_{s}(x) \in \Phi_{s}(x)$. We may find in [12] the fact that $u_{s}$ can be chosen in $L_{l o c}^{\infty}\left(\mathbb{R}^{n}\right)$. If the compact set $u_{s}\left(I_{c}\right)$ is not included in $\overline{\mathcal{B}^{m}}$, we choose with no loss of generality $\delta>0$ such that $u_{s}\left(I_{c}\right)$ is included in $\delta \overline{\mathcal{B}^{m}}$. Finally we have that $u_{s}$ is in $\dot{\mathcal{P}}(\Gamma)$. So the system (5) is weakly stabilizable under the feedback control $u_{s}$.

Besides, suppose that $V$ satisfies the small control property. It is shown in $[9$, Theorem 4.3] that we may extend $\Phi_{s}$ on $I_{c}$ by $\Phi_{s}(0)=\{0\}$ such that $\Phi_{s}$ is now lower semi-continuous on $I_{c}$. Applying the theorem 9 of Mickael, there exists $u_{s} \in \mathcal{P}(\Gamma)$ that stabilizes the system (5).

Remark 12 The feedback control $u_{s}: I_{c} \rightarrow \delta \overline{\mathcal{B}^{m}}$ is defined on the compact set $I_{c}$ which is attractive for the system, i.e. all solutions starting from $I_{c}$ stay in $I_{c}$. Moreover, it is bounded with values in $\delta \overline{\mathcal{B}^{m}}$.

\section{An explicit formula for the control law}

Let us adapt the Lin-Sontag universal formula for stabilization with bounded control :

Theorem 13 [13] If $V$ is a common control Lyapunov function for the system (5), then the feedback control

$$
u_{s}(x)= \begin{cases}-\frac{a_{s}(x)+\sqrt{a_{s}(x)^{2}+b_{s}(x)^{2}}}{b_{s}(x)\left(1+\sqrt{1+b_{s}(x)}\right)} B_{s}^{T}(x) & \text { if } x \neq 0 \\ 0 & \text { if } x=0\end{cases}
$$

belongs to $\dot{\mathcal{P}}(\Gamma)$ and weakly stabilizes the system (5). Moreover, if $V$ satisfies the small control property, then the feedback control (7) belongs to $\mathcal{P}(\Gamma)$, and thus the system (5) is stabilized.

Remark 14 If $V \in C^{k}\left(\mathbb{R}^{n} \backslash\{0\}, \overline{\mathcal{B}^{m}}\right)$ then $u_{s}$ is in $C^{k-1}\left(\mathbb{R}^{n} \backslash\{0\}, \overline{\mathcal{B}^{m}}\right)$.

In order to illustrate Theorem 13, we study an academical example, which can also be treated by using robust control strategy with uncertain parameters. This example shows the limits of the theoretical theorem 13.

Example 15 Let us consider the system

$$
\left\{\begin{array}{l}
\dot{x}_{1}=x_{1} x_{2} \\
\dot{x}_{2}=\frac{-x_{2}+u}{1+k}
\end{array}\right.
$$


where $k \in\{1,2,3,4\}, u \in \mathbb{R}$, and the common control Lyapunov function candidate

$$
V=\frac{x_{1}^{2}+\left(x_{1}^{2}+x_{2}\right)^{2}}{2} .
$$

We have

$$
\begin{aligned}
& a_{k}(x)=x_{1}^{2} x_{2}+\left(x_{2}+x_{1}^{2}\right)\left(2 x_{1}^{2} x_{2}-\frac{x_{2}}{1+k}\right), \\
& B_{k}(x)=\frac{x_{1}^{2}+x_{2}}{1+k} .
\end{aligned}
$$

It is obvious that $V$ is a common control Lyapunov function for the switched system over any compact set $I_{c}$ with $c>0$. Indeed, for $x \neq 0, B_{k}(x)=0$ implies $a_{k}(x)=-x_{1}^{4}<0$ and all the sets $I_{c}$ are invariant and attractive.

Also we have,

$$
a_{k}(x)=-\frac{x_{2}^{2}}{1+k}-2 x_{1}^{4} B_{k}(x)+2\left(x_{1} B_{k}(x)(1+k)\right)^{2} .
$$

It follows from Remark 10 that the small control property holds. So the system is stabilizable over all sets $I_{c}$ by using the following continuous bounded feedback control

$$
u_{k}(x)=\left\{\begin{array}{ll}
-\frac{a_{k}(x)+\sqrt{a_{k}(x)^{2}+b_{k}(x)^{2}}}{b_{k}(x)\left(1+\sqrt{1+b_{k}(x)}\right)} B_{k}^{T}(x) & \text { if } x \neq 0 \\
0 & \text { if } x=0
\end{array} .\right.
$$

This leads to the simulation on Fig.1.

\section{A constructive method for the common control Lyapunov func- tion}

In this section, we give a constructive method for a switched system globally defined where $\Gamma$ is a finite set of indexes.

Let us consider a family of dynamical systems :

$$
\dot{x}=f_{i}(x)+\left\langle g_{i}(x), u\right\rangle, \quad x \in \mathbb{R}^{n}, u \in \overline{\mathcal{B}^{m}}, i \in \Gamma
$$

where for all $i$ in $\Gamma, f_{i}, g_{i}$ are locally Lipschitz. This gives rise to the following switched system

$$
\dot{x}(t)=f_{s(t)}(x(t))+\left\langle g_{s(t)}(x(t)), u\right\rangle,
$$

where $s: \mathbb{R}_{\geq 0} \rightarrow \Gamma$ is a switching signal with finite values.

Let us introduce the notion of control Lyapunov function for the system (8). 


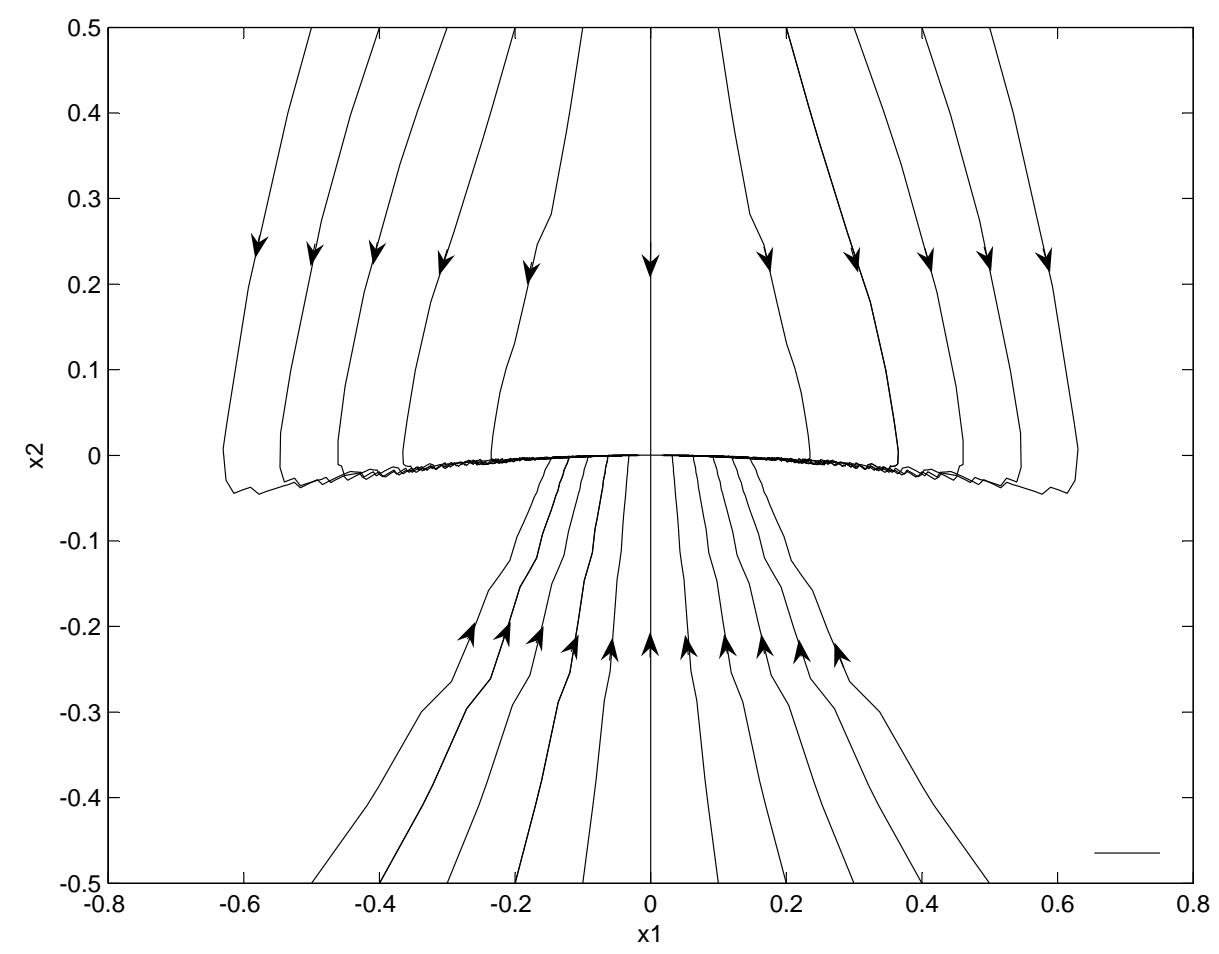

Fig. 1. Phase portrait of the closed-loop system

Definition 16 Let $i \in \Gamma$. A $C^{1}$ positive definite proper function $V_{i}: \mathbb{R}^{n} \rightarrow$ $\mathbb{R}_{\geq 0}$ is said to be a control Lyapunov function for the subsystem

$$
\dot{x}=f_{i}(x)+\left\langle g_{i}(x), u\right\rangle, \quad x \in \mathbb{R}^{n}, u \in \overline{\mathcal{B}^{m}}
$$

if for all $x \in \mathcal{V} \backslash\{0\}$,

$$
\min _{u \in \overline{\mathcal{B}^{m}}}\left\langle\nabla V_{i}(x), f_{i}(x)+\left\langle g_{i}(x), u\right\rangle\right\rangle<0 .
$$

As the function $u \rightarrow\left\langle\nabla V_{i}(x), f_{i}(x)+\left\langle g_{i}(x), u\right\rangle\right\rangle$ is continuous on the compact set $\overline{B^{m}}$, for all $x \in \mathcal{V}$ there exists a non-empty open set $\mathcal{U}_{i}(x) \subset \overline{\mathcal{B}^{m}}$ such that

$$
\left\langle\nabla V_{i}(x), f_{i}(x)+\left\langle g_{i}(x), u\right\rangle\right\rangle<0
$$

for all $u \in \mathcal{U}_{i}(x)$. In the following, we will use the notation $\mathcal{U}_{i}(x)$.

Now we give a criterion allowing to build a common control Lyapunov function, by using the control Lyapunov functions of the subsystems.

Criterion 17 Let a function $\gamma: \mathbb{R}^{n} \rightarrow \mathbb{R}$ of class $C^{1}$ be given such that $\gamma\left(\mathbb{R}_{\geq 0}^{n}\right) \subseteq \mathbb{R}_{\geq 0}$ and $\nabla_{j} \gamma\left(V_{1}(x), \ldots, V_{n}(x)\right)>0$. Suppose that we have the following assumptions :

(1) for each $i \in \Gamma, V_{i}: \mathbb{R}^{n} \rightarrow \mathbb{R}_{\geq 0}$ is a control Lyapunov function for the subsystem (9), 
(2) for all $i \in \Gamma$ and all $x \in \mathbb{R}^{n} \backslash\{0\}$, there exists $u_{0} \in \mathcal{U}_{i}(x)$ such that

$$
\begin{aligned}
& \sum_{\substack{j \in \Gamma \\
j \neq i}}\left\langle\nabla_{j} \gamma\left(V_{1}(x), \ldots, V_{n}(x)\right) \nabla V_{j}(x), f_{i}(x)+\left\langle g_{i}(x), u_{0}\right\rangle\right\rangle \\
& <-\left\langle\nabla_{i} \gamma\left(V_{1}(x), \ldots, V_{n}(x)\right) \nabla V_{i}(x), f_{i}(x)+\left\langle g_{i}(x), u_{0}\right\rangle\right\rangle
\end{aligned}
$$

then $V(x)=\gamma\left(V_{1}(x), \ldots, V_{n}(x)\right)$ is a common control Lyapunov function for the system (8), and the feedback control is given by :

$$
u_{i}(x)= \begin{cases}-\frac{a_{i}(x)+\sqrt{a_{i}(x)^{2}+b_{i}(x)^{2}}}{b_{i}(x)\left(1+\sqrt{1+b_{i}(x)}\right)} B_{i}^{T}(x) & \text { if } x \neq 0 \\ 0 & \text { if } x=0\end{cases}
$$

where :

$$
\begin{aligned}
a_{i}(x) & =\left\langle\nabla V(x), f_{i}(x)\right\rangle, \\
B_{i}(x) & =\left\langle\nabla V(x), g_{i}(x)\right\rangle \in \mathbb{R}^{m}, \\
b_{i} & =\left\|B_{i}(x)\right\|_{m}^{2} .
\end{aligned}
$$

Moreover, if $V$ satisfies the small control property then the feedback control (11) is continuous at the origin.

Proof. Let $x \in \mathbb{R}^{n}$ and $i \in \Gamma$. Suppose that there exists $\gamma$ satisfying the conditions of the theorem, we have :

$$
\begin{aligned}
& \left\langle\nabla V(x), f_{i}(x)+\left\langle g_{i}(x), u\right\rangle\right\rangle=\left\langle\sum_{j \in \Gamma} \nabla_{j} \gamma(\widehat{V}(x)) \nabla V_{j}(x), f_{i}(x)+\left\langle g_{i}(x), u\right\rangle\right\rangle \\
& =\left\langle\nabla_{i} \gamma(\widehat{V}(x)) \nabla V_{i}(x), f_{i}(x)+\left\langle g_{i}(x), u\right\rangle\right\rangle+\sum_{\substack{j \in \Gamma \\
j \neq i}}\left\langle\nabla_{j} \gamma(\widehat{V}(x)) \nabla V_{j}(x), f_{i}(x)+\left\langle g_{i}(x), u\right\rangle\right\rangle
\end{aligned}
$$

with $\widehat{V}(x)=\left(V_{1}(x), \ldots, V_{n}(x)\right)$.

By using assumption (2), we know that there exists $u_{0} \in \mathcal{U}_{i}(x)$ such that

$$
\sum_{j \in \Gamma}\left\langle\nabla_{j} \gamma\left(V_{1}(x), \ldots, V_{n}(x)\right) \nabla V_{j}(x), f_{i}(x)+\left\langle g_{i}(x), u_{0}\right\rangle\right\rangle<0 .
$$

So, we have, for all $x \in \mathbb{R}^{n} \backslash\{0\}$,

$$
\min _{u \in \overline{\mathcal{B}^{m}}}\left\langle\nabla V(x), f_{i}(x)+\left\langle g_{i}(x), u\right\rangle\right\rangle<0 .
$$

Thus $V$ is a common control Lyapunov function for the system (8).

So, using Theorem 13, we know that the feedback control (11) stabilizes the system (8). 
For example

$$
\gamma(x)=\sum_{i \in \Gamma} x_{i}
$$

leads to

$$
V(x)=\sum_{i \in \Gamma} V_{i}(x)
$$

Remark 18 If we choose

$$
V(x)=\min _{i \in \Gamma} V_{i}(x)
$$

the common control Lyapunov function is not differentiable. Using such a common control Lyapunov function leads to introduce the proximal subdifferential of $V$. Then, there exists a condition on the proximal subdifferential equivalent to condition (4). So a feedback control can be built but it is discontinuous. For more details on this point, the reader can see [14].

In order to illustrate Criterion 17, we now propose to study the following example.

Example 19 Let us consider the system that switches between the two subsystems :

$$
\left\{\begin{array}{l}
\dot{x_{1}}=-x_{1}+x_{2} \\
\dot{x_{2}}=x_{2}+u
\end{array}\right.
$$

and

$$
\left\{\begin{array}{l}
\dot{x_{1}}=-x_{2} \\
\dot{x_{2}}=x_{1}+u x_{2}
\end{array} .\right.
$$

The two candidate control Lyapunov functions are $V_{1}(x)=x_{1}^{2}+x_{2}^{2}$ for $\left(S_{1}\right)$ and $V_{2}(x)=\frac{3}{2} x_{1}^{2}+x_{2}^{2}-2 x_{1} x_{2}$ for $\left(S_{2}\right)$.

Given $(i, j) \in\{1,2\}^{2}$, let us introduce the following notations :

$$
\begin{aligned}
a_{i j}(x) & =\left\langle\nabla V_{i}(x), f_{j}(x)\right\rangle, \\
B_{i j}(x) & =\left\langle\nabla V_{i}(x), g_{j}(x)\right\rangle,
\end{aligned}
$$

where $f_{j}$ and $g_{j}$ are the components of the subsystem $S_{j}$. We have:

$$
\begin{array}{ll}
a_{11}(x)=-2 x_{1}^{2}+2 x_{2}^{2}+2 x_{1} x_{2}, & B_{11}(x)=2 x_{2}, \\
a_{21}(x)=3 x_{1}\left(x_{2}-x_{1}\right), & B_{21}(x)=2\left(x_{2}-x_{1}\right), \\
a_{12}(x)=0, & B_{12}(x)=2 x_{2}^{2}, \\
a_{22}(x)=-2 x_{1}^{2}-x_{1} x_{2}+2 x_{2}^{2}, & B_{22}(x)=2 x_{2}\left(x_{2}-x_{1}\right) .
\end{array}
$$

For all $i \in\{1,2\}$ and all $x \neq 0, B_{i i}(x)=0$ implies that $a_{i i}(x)<0$, which shows that $V_{i}$ is a control Lyapunov function for the subsystem $\left(S_{i}\right)$. As $a_{12}=0$ 
and as $B_{21}(x)=0$ implies that $a_{21}(x)=0$, we can deduce that for $i \neq j, V_{i}$ is not a control Lyapunov function for the subsystem $\left(S_{j}\right)$.

We now check the conditions of the criterium 17 with the function $\gamma(x)=$ $x_{1}+x_{2}$ to build our common control Lyapunov function. So we must verify that for $x \in \mathbb{R}^{2} \backslash\{0\}, a_{11}(x)+a_{21}(x)<0$ when $b_{11}(x)+b_{21}(x)=0$ and $a_{22}(x)+a_{12}(x)<0$ when $b_{22}(x)+b_{12}(x)=0$. This leads to

- $b_{11}(x)+b_{21}(x)=4 x_{2}-2 x_{1}=0$ for $x_{1}=2 x_{2}$, which implies that $a_{11}(x)+$ $a_{21}(x)=-6 x_{2}^{2}$, which is strictly negative.

- $b_{22}(x)+b_{12}(x)=2 x_{2}\left(2 x_{2}-x_{1}\right)=0$ for $x_{2}=0$ or $x_{1}=2 x_{2}$, which implies that if $x_{2}=0, a_{22}(x)+a_{12}(x)=-2 x_{1}^{2}$ and for $x_{1}=2 x_{2}, a_{22}(x)+a_{12}(x)=-8 x_{2}^{2}$. So the condition is checked.

Thus, the function $V(x)=V_{1}(x)+V_{2}(x)$ is a common control Lyapunov function for the switched system over any compact set $I_{c}$ with $c>0$. The feedback control (11) stabilizes the switched system on each set $I_{c}$ and it is bounded. The figure 2 represents the phase portrait of the closed-loop system, with the function $t \mapsto \sin (t)$ as switching signal.

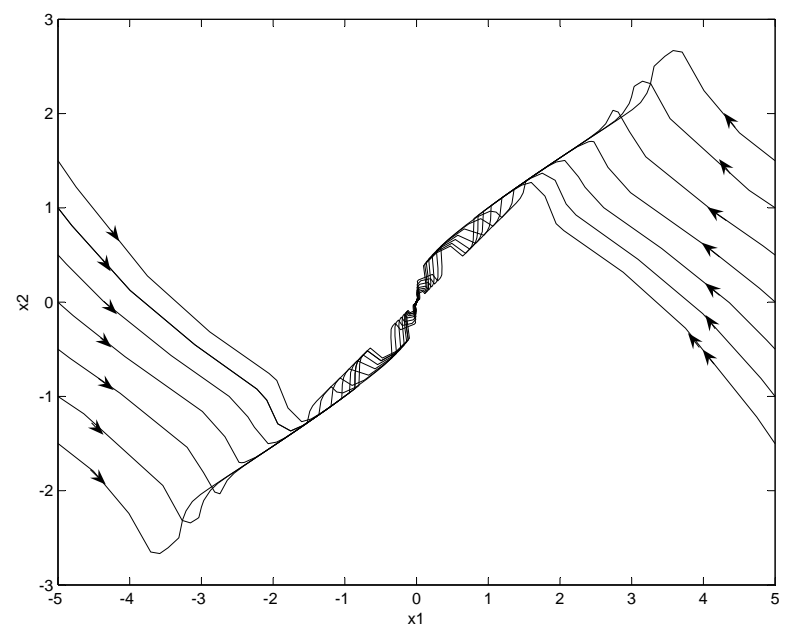

Fig. 2. Phase portrait of the closed-loop system

\section{Conclusion}

In this paper, we first emphasize a necessary and sufficient condition for the stabilization of nonlinear switched systems, by using the notion of common control Lyapunov function. Then we build an explicit feedback control by using the Lin-Sontag formula. Nevertheless, in practical, it is quite difficult to find such a function. This is the reason why we develop a practical criterion in order to build a common control Lyapunov function from a family of control Lyapunov functions attached to the subsystems. 
Our feedback control does not depend of the $C$-switching signal, which is a severe condition. This implies that the class of systems studied is restricted. For future directions, we will try to develop a control law for a given $C$-switching signal which can be adapted to a larger class of systems.

\section{References}

[1] D. Liberzon, A. Morse, Basic problems in stability and design of switched systems, IEEE Control Systems Mag. (1999) 59-70.

[2] J. Malmborg, Analysis and design of hybrid control systems, Ph.D. thesis, Lund Instotute of Technology, Sweden (1998).

[3] S. Pettersson, B. Lennartson, Stability and robustness for hybrid systems, Proceedingsof the 35th Conference on Decision and Control (1996) 1202-1207.

[4] M. Branicky, Multiple lyapunov functions and other analysis tools for switched and hybrid systems, IEEE Transactions on Automatic Control 43 (4) (1998) 475-482.

[5] J. L. Mancilla-Aguilar, R. Garcia, E. Sontag, Y. Wang, On the representation of switched systems with inputs by perturbed control systems, Nonlinear Analysis: Theory, Methods \& Applications 60 (2005) 1111-1150.

[6] J. Mancilla-Aguilar, R. García, A converse Lyapunov theorem for nonlinear switched systems, Systems \& Control Letters 41 (2000) 67-71.

[7] L. Vu, D. Liberzon., Common lyapunov functions for families of commuting nonlinear systems, Systems \& Control Letters 54 (2005) 405-416.

[8] H. Sun, J. Zhao, Control lyapunov functions for switched control systems, in: Proccedings of the Amrican Control Conference, Arlington, VA, 2001, pp. 18901891.

[9] Z. Artstein, Stabilization with relaxed controls, Nonlinear Analysis: Theory, Methods \& Applications 7 (11) (1983) 1163-1173.

[10] J. P. Aubin, H. Frankowska, Set-Valued Analysis, Springer-Verlag, New-York, 1990.

[11] E. Mickael, Continuous selections., Anal. Math. 63 (2) (1956) 361-382.

[12] L. Rifford, On the existence of nonsmooth control-lyapunov functions in the sense of generalized gradients 6 (2001) 593-611.

[13] Y. Lin, E. Sontag, A universal formula for stabilization with bounded controls, Systems \& Control Letters 16 (1991) 393-397.

[14] L. Rifford, Semiconcave control-lyapunov functions and stabilizing feedbacks, SIAM J. Control Optim. 41 (3) (2002) 659-681. 